\title{
A Radio-Frequency Permeameter
}

\author{
Peter H. Haas
}

\begin{abstract}
An instrument is described, which, in connection with commercially available impedance measuring devices, is capable of measuring the permeability and losses in ferromagnetic toroidal cores. The measurement is made by inserting a toroidal core into the short-circuited secondary of a transformer whose primary is attached to a radio-frequency bridge or $Q$ meter. Equations are derived for the measurements, the accuracy and sensitivity are discussed, and typical construction details are given. Finally, a modification of the device enabling the measurement of permeability temperature coefficients is discussed.
\end{abstract}

\section{Definition of Symbols}

$L_{1}=$ Input inductance with the top cover removed.

$C_{1}=$ Capacitance read on a $Q$ meter, admittance bridge or parallel condenser with the top cover removed.

$L_{0}=$ Input inductance with the top cover shorting the secondary.

$C_{0}=$ Capacitance with the top cover shorting the secondary.

$L_{f}=$ Input inductance with the test sample inserted.

$C_{f}=$ Capacitance with the test sample inserted.

$R_{1}, R_{0}, R_{f}=$ Series resistances corresponding to the above conditions.

$G_{1}, G_{0}, G_{f}=$ Parallel conductances corresponding to the conditions.

$Q_{1}, Q_{0}, Q_{f}=$ Values of the storage factor $Q$ read on a $Q$ meter corresponding to the above conditions.

$L_{10}, R_{01}, C_{01}=-L_{1}-L_{0}, R_{0}-R_{1}, C_{0}-C_{1}$, respectively.

$L_{1 f}, R_{f 1}, C_{f 1}=L_{1}-L_{f}, R_{f}-R_{1}, C_{f}-C_{1}$, respectively.

$L_{f 0}, C_{0 f}=L_{f}-L_{0}$ and $C_{0}-C_{f}$, respectively.

$V_{1}=$ Voltage applied across the primary terminals of the permeameter.

$e_{0 c 2}=$ Voltage appearing across the open-circuited secondary with $V_{1}$ across the primary.

$\mu_{0}^{\prime}=$ Initial permeability.

$\tan \delta_{m}=$ Magnetic dissipation factor. Note: $\left.\tan \delta_{m} / \mu_{0}^{\prime}=1 / \mu_{0}^{\prime} Q\right)$.

The symbols $R$ and $L$ used in the introduction denote the equivalent series resistance and inductance of a core, respectively.

The units used are those defined in the rationalized MKS system, i. e., henries, farads, ohms, and mhos, for inductance, capacitance, resistance, and conductance, respectively.

\section{Introduction}

With increasing use of magnetic cores at radio frequencies coupled with more stringent demands in communications equipment, it has become more important to accurately evaluate the behavior of core materials in the frequency and temperature range in which they are to be used. At the same time, mass-production methods demand a simple and rapid check for quality-control purposes. In addition to these requirements, some of the newer materials, with their wide spread of permeabilities, have made older testing methods either inaccurate or insensitive. The instrument described was designed to fill the need for a quick testing method of moderate accuracy over the greater part of the range of permeabilities currently available at radio frequencies below $20 \mathrm{Mc} / \mathrm{s}$. With proper caution, this frequency limit may well be exceeded.
It is desired to measure the complex initial permeability

$$
\mu_{0}^{*}=\mu_{0}^{\prime}-j \mu_{0}^{\prime \prime},
$$

where $\mu_{0}^{\prime}$ is the quantity usually called simply the initial permeability, and $\mu_{0}^{\prime \prime} / \mu_{0}^{\prime}$ is the initial dissipation factor, $\tan \delta_{m}$. Initial permeability is that value of $\mu^{\prime}$ measured on a previously demagnetized sample with such low field strengths that no change is noticed when the field is reduced still further. The initial dissipation factor $\tan \delta_{m}$ is measured under the same conditions. Ideally, it therefore does not contain any losses caused by hysteresis and its resistive component is not a function of the field. It may be noted that Legg's loss factor, $L,[1]^{1}$ equals $^{2}$

$$
\frac{R}{\mu_{0}^{\prime} f L}=\frac{2 \pi \tan \delta_{m}}{\mu_{0}^{\prime}}
$$

In the past many laboratories have used a method of measurement in which toroidal cores have a number of turns of wire placed upon them. An impedance bridg*e or $Q$ meter is then used to measure the effective inductance and resistance, and the permeability is calculated from these values and the known geometry of the core. When all the losses not contributed by the core are deducted, one can also calculate the dissipation factor. This method, while cumbersome because of the labor involved in the toroidal winding process, is accurate enough if all the factors contributing to errors are taken into consideration. Some of these factors are:

1. At higher frequencies it is necessary to reduce the number of turns to a minimum, so that the reactance of the ring will fall within the measurable reactance range of an impedance bridge or within the capacitance range of a $Q$ meter. This, in the case of low- and medium-permeability materials, leads to an error because of violation of the uniformcurrent-sheet assumption.

2. Some of the newer core materials, especially those with high permeabilities, are known to exhibit high dielectric constants as well as high dielectric losses. If the product of permeability and dielectric

\footnotetext{
1 Figures in brackets indicate the literature refercnces at the end of this paper. 2 See appendix III for definitions of symbols.
} 
constant is sufficiently high, a large percentage of a wavelength may exist even at low frequencies in a dimensionally small core. The errors caused by this effect can be very large. This case and the correction of the errors involved are admirably well discussed in the literature [2]. There is, however, even for fairly low dielectric constants, the additional problem caused by displacement currents between windings separated by the core material. This causes the inductance that would actually be present if the material had unity permeability and the dielectric constant found in a ferromagnetic core to differ from the inductance $L_{a}$ (see appendix II) calculated from the core dimensions and the number of turns.

A simple method used primarily in the powderediron fields yields the effective permeability, $\mu_{\text {eff }}$, and dissipation factor, $\tan \delta_{\text {eff }}$, of slug's. One simply notes the change of inductance or resonating capacitance caused by the insertion of a rod into a standard coil. Although it is sometimes possible to evaluate the true material constants $\mu_{0}^{\prime}$ and $\tan \delta_{m}$, given the length to diameter ratio, this ratio must be unreasonably large to obtain satisfactory sensitivity for permeabilities of several hundred and higher.

A third method used at the National Bureau of Standards and other laboratories to obtain the highest accuracies involves the use of thin annular disks accurately ground or machined from the core material. These are inserted into a coaxial line whose impedance changes are measured. From the viewpoint of accuracy this is a highly satisfactory method. The aforementioned errors either do not apply or their elimination or correction is relatively simple. However, the method suffers from a number of drawbacks. As mentioned above, the sample must be prepared with the highest precision. The test is destructive insofar as the sample is not usually of a shape usable in application. Furthermore, special, low-impedance measuring instruments are often required for the measurements.

\section{Description of the Radio-Frequency Permeameter}

Inasmuch as the requirements for an accurate coaxial line measurement are somewbat stringent, there exists a need for a method of lesser accuracy for use in conjunction with commercially available measuring instruments. The test samples employed should be readily available without any need for accurate shaping and ideally, ought to be usable in applications. It is believed that the method here described meets these requirements. In 1924, G. A. Kelsall developed an instrument to measure the permeability of core materials at power-]ine and low audiofrequencies [3]. The RF permeameter (fig. 1) described in this paper is essentially of the same construction. Certain changes were made, necessitated by the higher frequencies used. The complete equations, taking losses into consideration, were developed to allow the measurement of the

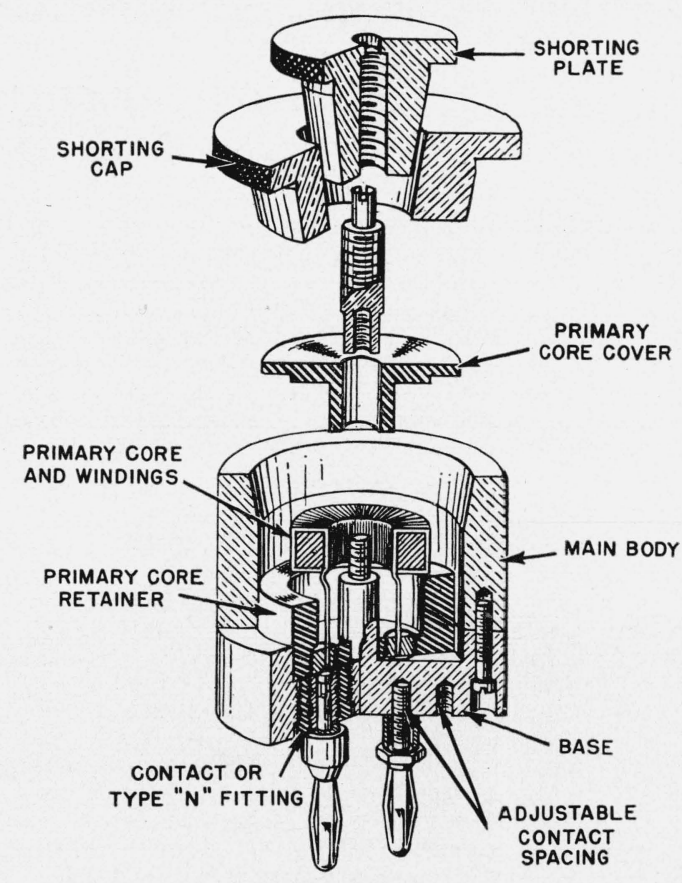

Figure 1. Cutaway view of radio-frequency permeameter with transformer.

radio-frequency losses in core materials. ${ }^{3}$ Additional changes were made to adapt the instrument to the measurement of temperature coefficients of permeability.

\section{Analysis}

The transformer is represented by the network shown in figure 2 . Then the input impedance, $Z_{\text {in }}$ at the primary terminals for three conditions in the secondary is

$$
Z_{1}=R_{1}+j \omega L_{1}
$$

for the secondary open-circuited,

$$
Z_{0}=R_{1}+j \omega L_{1}+\frac{\omega^{2} M^{2}}{R_{2}+j \omega L_{2}}
$$

for the secondary short-circuited without sample inserted,

$$
Z_{f}=R_{1}+j \omega L_{1}+\frac{\omega^{2} M^{2}}{\left(R_{2}+R_{u}\right)+j \omega\left(L_{2}+L_{u}\right)}
$$

when the short-circuited secondary impedance has been increased by a quantity $R \mu+j \omega L_{u}$ because of the insertion of a ferromagnetic core.

The use of a high $Q$ secondary allows us to make the following assumptions: ${ }^{4}$

\footnotetext{
${ }^{3}$ At present it appears impossible to use the method described herein for the determination of values of $\mu^{\prime \prime}<0.1$ approximately.

4 See appendix I.
} 


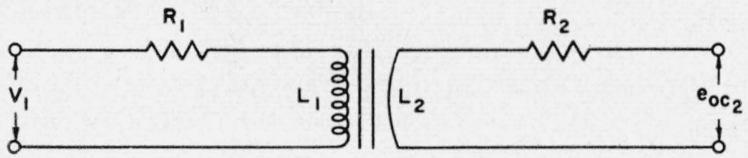

Figure 2. Transformer representation.

$$
\begin{gathered}
R_{2}^{2} \ll \omega^{2} L_{2}^{2} \\
\left(R_{2}+R_{u}\right)^{2} \ll \omega^{2}\left(L_{2}+L_{u}\right)^{2} .
\end{gathered}
$$

In the above equations the mutual inductance, $M$, which is not directly measurable from the input terminals, must be derived.

We note that if the secondary be left open-circuited and a voltage, $V_{1}$, be applied to the primary terminals,

$$
\begin{gathered}
V_{1}=\left(R_{1}+j \omega L_{1}\right) i_{1} \\
e_{0 c_{2}}=j \omega M i_{1},
\end{gathered}
$$

and

$$
\frac{\left|V_{1}\right|}{\left|e_{0 c_{2}}\right|}=\frac{\sqrt{R_{1}^{2}+\omega^{2} L_{1}^{2}}}{\omega M}
$$

The primary coil is selected to have a specially high $Q$, so that

$$
R_{1}^{2} \ll \omega^{2} L_{1}^{2}
$$

We therefore have

$$
\frac{\left|V_{1}\right|}{\left|e_{0 c_{2}}\right|}=\frac{L_{1}}{M}
$$

For convenience, let this ratio be defined

$$
\frac{\left|V_{1}\right|}{\left|e_{0 c_{2}}\right|}=N \text {. }
$$

If the leakage inductance is negligible, this is exactly equal to the turns ratio, and for moderate accuracies, this assumption may often be made. We shall discuss this point later in some detail.

Using these conditions we may solve eq (2) and (3) for $R_{2}, L_{2}, R_{u}$ and $L_{u}$ respectively.

$$
\begin{aligned}
Z_{0}-Z_{1} & =R_{2}\left(\frac{L_{1}}{N L_{2}}\right)^{2}-j \frac{\omega}{L_{2}}\left(\frac{L_{1}}{N}\right)^{2} \\
L_{2} & =\left(\frac{L_{1}}{N}\right)^{2} \frac{1}{L_{1}-L_{0}}=\left(\frac{L_{1}}{N}\right)^{2} \frac{1}{L_{10}} \\
R_{2} & =\left(\frac{L_{1}}{N}\right)^{2} \frac{R_{0}-R_{1}}{\left(L_{1}-L_{0}\right)^{2}}=\left(\frac{L_{1}}{N}\right)^{2} \frac{R_{01}}{L_{10}^{2}},
\end{aligned}
$$

where

$$
L_{10}=L_{1}-L_{0}
$$

Furthermore,

$$
R_{01}=R_{0}-R_{1}
$$

$$
\begin{aligned}
& Z f-Z_{1}=\frac{\left(\frac{\omega L_{1}}{N}\right)^{2}}{\left(R_{2}+R_{u}\right)+j \omega\left(L_{2}+L_{u}\right)} \\
& R_{u}=\left(\frac{L_{1}}{N}\right)^{2}\left[\frac{R_{f 1}}{L_{1} f^{2}}-\frac{R_{01}}{L_{10}^{2}}\right] \\
& L_{u}=\left(\frac{L_{1}}{N}\right)^{2} \frac{L_{f 0}}{L_{1 f} L_{10}},
\end{aligned}
$$

where

$$
R_{f 1}=R_{f}-R_{1} ; \quad L_{f 0}=L_{f}-L_{0} ; \quad L_{1 f}=L_{1}-L_{f} .
$$

We have thus been able to solve for the added inductance and resistance arising from the presence of the ferromagnetic test core in terms of the measured values $R_{1}, L_{1}, L_{0}, R_{0}, R_{f}$, and $L_{f}$. It remains then to calculate the permeability, $\mu^{\prime}$, and the dissipation factor, $\tan \delta_{m}$, from these values.

The value of $L_{u} /\left(\mu^{\prime}-1\right)$ may be calculated from one of the available formulas (see appendix II). Let this value be called $L_{a}$. Thus $\mu^{\prime}-1=L_{u} / L_{a}$ and

$$
\mu^{\prime}-1=\frac{\left[\left(\frac{L_{1}}{N}\right)^{2} \frac{1}{L_{10}}\right] \frac{L_{f 0}}{L_{1 f}} .}{L_{a}} .
$$

Furthermore, since

$$
\frac{R_{u}}{\omega L_{u}}=\tan \delta_{m},
$$

$$
\tan \delta_{m}=\frac{L_{10} L_{1 f}}{\omega L_{f 0}}\left[\frac{R_{f 1}}{L_{1 f}^{2}}-\frac{R_{01}}{L_{10}^{2}}\right]
$$

It is noteworthy that the bracketed quantity in eq (20) is a constant that may be calibrated. Similarly the last ratio in the brackets and $L_{10}$ in eq (22) are constants. Thus only the differences $L_{f}-L_{0}, L_{1}-$ $L_{f}$, and $R_{f}-R_{1}$ need to be measured for each test core.

The equations (20) and (22) give $\mu^{\prime}$ and $\tan \delta_{m}$ in terms of series components of resistance and inductance obtainable with a radio-frequency bridge. The extension to other instruments, such as an admittance bridge reading parallel components of conductance and capacitance, or to a $Q$ meter is immediate. The equations are:

(A) Admittance bridge, reading $G+j \omega C$ :

$$
\left(\mu^{\prime}-1\right)=\frac{\left[\frac{1}{\omega^{2} N^{2} C_{01}}\right] \frac{C_{0 f}}{C_{f 1}} .}{L_{a}}
$$

$\tan \delta_{m}=\frac{C_{01} C_{f 1}}{\omega C_{0 f}}\left[\frac{1}{C_{f 1}^{2}}\left(G_{f}-\frac{C_{f}^{2}}{C_{1}^{2}} G_{1}\right)-\frac{1}{C_{01}^{2}}\left(G_{0}-\frac{C_{0}^{2}}{C_{1}^{2}} G_{1}\right)\right]$. 
(B) $Q$ meter, reading resonating capacitance $C$, and Q:

$\mu^{\prime}-1$, same as eq (23)

$$
\left(\mu^{\prime}-1\right)=\frac{\left[\frac{1}{\omega^{2} N^{2} C_{01}}\right] \frac{C_{0 f}}{C_{f 1}},}{L_{a}},
$$

$\tan \delta_{m}=\frac{C_{01} C_{f 1}}{C_{0 f}}\left[\frac{1}{C_{f 1}^{2}}\left(\frac{C_{f}}{Q_{f}}-\frac{C_{f}^{2} C_{1}}{C_{1}^{2} Q_{1}}\right)-\frac{1}{C_{01}^{2}}\left(\frac{C_{0}}{Q_{0}}-\frac{C_{0}^{2} C_{1}}{C_{1}^{2} Q_{1}}\right)\right]$.

The choice of instruments will depend on the frequency range to be covered, the desired accuracy and sensitivity, as well as on the construction of the permeameter and its primary transformer. An additional method, capable of high sensitivity in the value of the permeability, especially for the measurement of temperature coefficients, of permeability, uses a radiofrequency impedance bridge, such as General Radio type $916 \mathrm{AL}$, in parallel with a variable capacitor, such as General Radio type $722 \mathrm{~N}$. The bridge is balanced with the permeameter connected to its unknown terminals in parallel with the capacitor. The balance is made with the secondary open-circuited and the capacitor set at its lowest value. The rest of the measurements are made as before, except that the reactive portion of the balance is made with the parallel capacitor only. The permeability is given by eq (23), and

$$
\begin{array}{r}
\tan \delta_{m}=\frac{R_{p}}{\omega^{2} L_{1}^{2}} \frac{C_{01} C_{f 1}}{\omega C_{0 f}}\left\{\frac{1}{C_{f 1}^{2}}\left[\frac{R_{f}}{R_{1}}-\left(1+\omega^{2} L_{1} C_{f 1}\right)^{2}\right]\right. \\
\left.-\frac{1}{C_{01}^{2}}\left[\frac{R_{0}}{R_{1}}-\left(1+\omega^{2} L_{1} C_{01}\right)^{2}\right]\right\} .
\end{array}
$$

An additional measurement that needs to be made for the evaluation of $\tan \delta_{m}$ is one for the primary resistance, $R_{p}$, which must be made without the parallel capacitor.

\section{Details of Measurements}

In general, the measurements are performed in the same manner as any other impedance measurement. Certain precautions, however, will increase the accuracy. Upon examination of eq (20) through (27), it will be noted that in most cases only differences of impedance are required. Many of the bridges used, as well as the $Q$ meter, are provided with vernier capacitors, which may be used to advantage. The short-circuited impedance reading is usually close to the bridge reading with the sample inserted when the latter has low permeability. For highpermeability samples, the difference between the open-circuited impedance reading and that with the sample inserted is usually small. Arranging the order of readings in proper sequence so that a main dial need not be moved, the entire difference reading being taken on the vernier capacitor, usually will increase the accuracy above the quoted accuracy of the bridge. For bridges of the series-impedance type, several transformers are necessary to cover a wide frequency range.

For the measurement of initial permeability and losses, extremely low fields are necessary. In his original article, Kelsall derived the expression for the magnetic field in the sample at the mean radius. The equation shows the field to be proportional to the number of turns on the primary and the total primary current and inversely proportional to the mean diameter, $D$, of the test core [4].

$$
H=\frac{2 N_{1}\left(L_{1}-L_{f}\right) I}{5 L_{1} D},
$$

where $H$ is in oersteds, and when $I$ is in amperes. For a constant primary voltage, however, the total current is inversely proportional to the input impedance, which is largely inductive. As the primary inductance itself is proportional to $N_{1}^{2}$, the field will be inversely proportional to $N_{1}$, the frequency, and the mean diameter, and proportional to the applied voltage. The voltage in practice can be made very low by using sensitive bridge detectors. Thus it can be seen that the conditions favor low magnetic fields and that it is not difficult to make a proper measurement of the initial permeability. In addition to facilitating this measurement, a low voltage is also desirable from the viewpoint of transformer stability and high-input $Q$ because of the reduction of hysteresis losses in the primary circuit. Needless to say, a high $Q$ is very desirable, especially for the measurement of dissipation factor.

\section{Accuracy}

It is difficult to determine the exact accuracy of the permeameter because too many indeterminate factors are involved. We shall discuss some of these to arrive at some estimate of the highest attainable accuracy and also mention some of the shortcomings of the instrument.

The problem of dimensional resonance caused by a high dielectric constant is, of course, not remedied in this instrument any more than if toroidal windings were placed on the core. The permeability and losses measured will simply be the effective values for that particular core. There are, however, only completely negligible disturbing effects caused by capacitance between turns and resulting displacement currents.

The accuracy of the bridge or of the $Q$ meter used will of course be a large factor in the over-all accuracy of the measurement. Obviously all of the corrections to the readings as specified by the instructions for the particular instrument have been made and care has been taken to keep all shunting capacitances at a minimum. By applying such corrections with care, 
it is easily possible to secure results that are more accurate than the rated accuracy of the instrument when this is used for a single measurement. This is because in most of the measurements only differences of reactance, resistance, or capacitance are required. It is rarely the case that the amount by which a bridge reading will be inaccurate will change drastically between two closely related readings. Unless the particular instrument is equipped with a vernier scale, the lack of large differences between readings will usually be a greater problem than lack of accuracy. The over-all accuracy can be improved by evaluation through a calibration experiment of that portion of the equation that is theoretically a constant. This point will be discussed in a later section.

Equation (26) contains both $Q_{1}$ and $Q_{f}$, which may differ by little for low-loss samples. If $Q_{1}$ is large, a more precise determination of this difference will be possible. The same advantage is implied in the other equations for the dissipation factor.

In order to get some estimate of the accuracy of the measurements, a number of samples having permeabilities between 20 and 850 were tested by ballistic methods by the Magnetic Measurements Section of the National Bureau of Standards. Although these measurements were made with direct current, they afford some comparison with the values obtained at $100 \mathrm{kc} / \mathrm{s}$ on the permeameter, as there is rarely much change of the permeability in that frequency range. The differences between the two methods ranged from 1.6 to 2.8 percent. Some comparisons, especially for the values of the dissipation factor, were also made with values obtained on coaxial samples of presumably the same materials. Because of the variations present from batch to batch and at times even in the same piece, this comparison is not altogether conclusive. It may be said, however, that where the permeabilities agreed to better than 5 percent, the agreement between the dissipation factors remained within 10 percent. It is believed that these are reasonably reliable values for the maximum accuracy, provided all possible care has been taken.

The frequency range within which the permeameter may be used is most limited by the decrease in sensitivity and also to some extent by the decreasing accuracy of measuring instruments. Good results have been obtained at the Bureau up to $20 \mathrm{Mc} / \mathrm{s}$. By proper design it should be possible to extend the upper limit.

\section{Calibration of the Permeameter}

\subsection{Voltage Ratio}

It is mentioned earlier in connection with eq (10) that the voltage ratio $\left|V_{1}\right| /\left|e_{0 c_{2}}\right|$ may be replaced by the turns ratio $N$ if the primary leakage inductance is negligible. This is essentially the case with transformer cores small by comparison to the radial dimensions of the instrument, of medium permeability ( $\mu^{\prime}=60$ or higher), and for turns ratios of the order
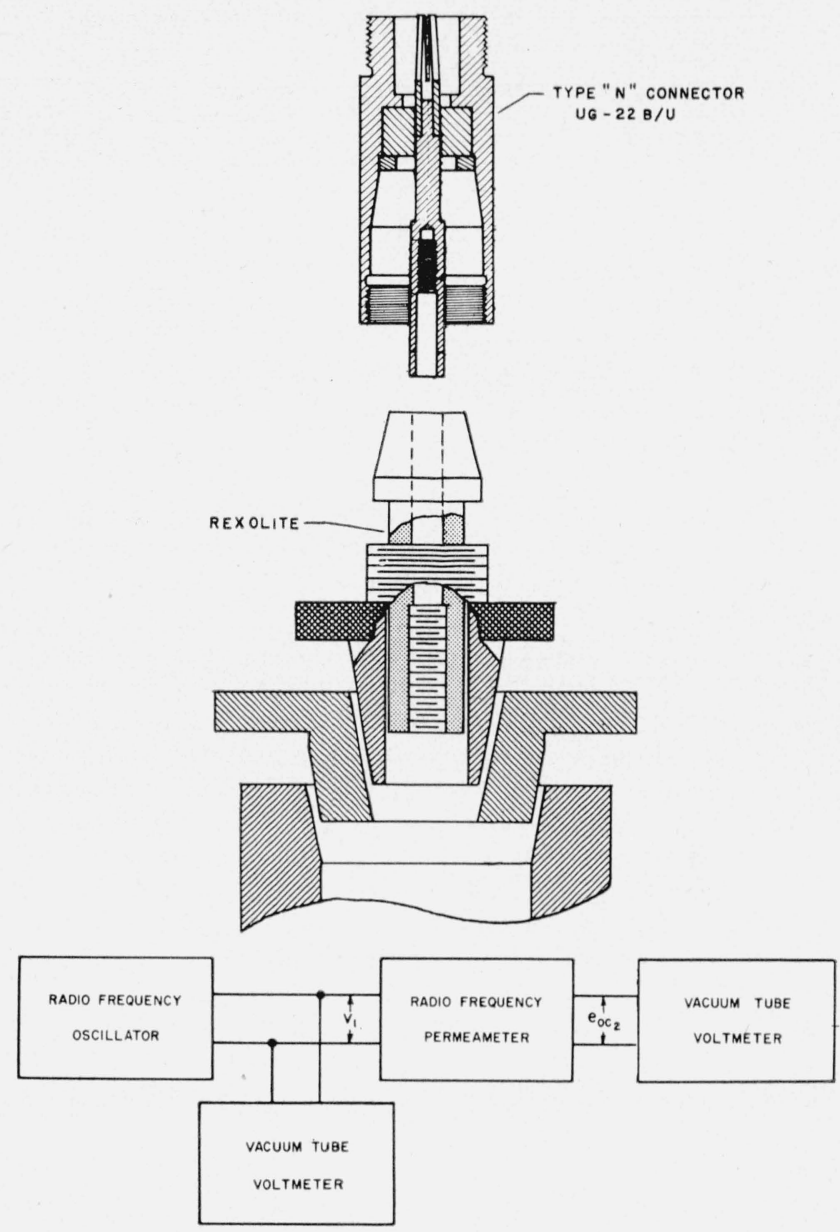

FIGURE 3. Experimental arrangement for measuring voltage ratio.

of 100 or greater. It has been found desirable, however, to use low-permeability cores ( $\mu^{\prime}$ below 20) for the higher frequency ranges because of the decrease in transformer losses. For best results, it is, therefore, desirable to measure the voltage ratio at several frequencies. For moderate accuracies, a good vacuum-tube voltmeter with a low voltage range (0.01or at most $0.1-v$ full scale) will suffice. The experimental arrangement is shown in figure 3, which also shows a top-connection plate replacing the topshorting plate (shown at the top of fig. 1) for this purpose. A stable radio-frequency generator with a voltage output approximately equal to that used for the permeability measurements is connected to the primary terminals, with provision for a shunting voltmeter close to the terminals. The voltage $V_{1}$ is read, and the voltmeter is then connected to the secondary terminals for the reading of $e_{0 c}$. It was found that for input coils with $Q$ of 50 or better over the entire frequency range, the ratio will remain constant within 5 percent from $100 \mathrm{kc} / \mathrm{s}$ to $5 \mathrm{Mc} / \mathrm{s}$, increasing at the upper limit. A few cases have been 

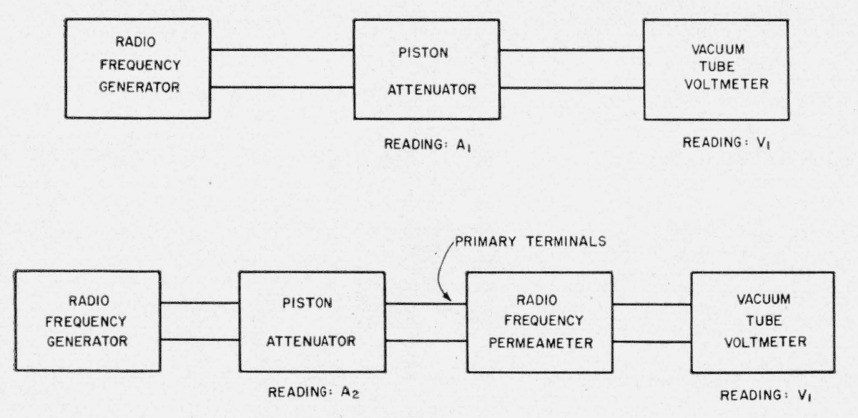

$$
\frac{\left|V_{1}\right|}{\left|e_{o c_{2}}\right|}=\operatorname{LOG}_{10}^{-1} \frac{A_{1}-A_{2}}{20}
$$

FIGURE 4. Calibration arrangement, using piston attenuator.

noted in which the deviations did not exceed 1 percent. It should be kept in mind that this ratio enters the equations as a square, therefore any error in this ratio will cause an error twice as large in the permeability measured.

A more accurate calibration method is shown in figure 4. The piston attenuator is adjusted so that the voltmeter reads a voltage $V_{1}$. The permeameter is then inserted between the attenuator and the voltmeter, and the attenuation is reduced until the voltmeter reads a voltage $V_{1}$ once more. If the attenuator readings were $A_{1}$ and $A_{2} \mathrm{db}$, respectively, the voltage ratio is given by

$$
\frac{\left|V_{1}\right|}{\left|e_{0 c_{2}}\right|}=\log _{10}{ }^{-1} \frac{A_{1}-A_{2}}{20} .
$$

This method is used at the National Bureau of Standards in conjunction with a standard piston attenuator for calibration of the permeameters. A dissipative attenuator, operating under matched conditions and with sufficient initial attenuation inserted, may also be used with somewhat reduced accuracy. Such an instrument should then have a dial calibrated in hundredths of decibels at least.

\subsection{Secondary Inductance}

Consider the expression

$$
\frac{1}{\omega^{2} N^{2} C_{01}}
$$

which appears as the multiplier of the ratio of the differences in capacitance in eq (23). We note that this is theoretically a constant for a particular permeameter independent of frequency or number of primary turns.

If we define $L_{1}=N^{2} \mu_{1} L_{a 1}$, where $L_{a 1}$ is the geometrical inductance of the primary core and $\mu_{1}$ its permeability, it can be shown that

$$
\begin{aligned}
\frac{1}{\omega^{2} C_{01} N^{2}} & =L_{2}-\mu_{1} L_{a 1} \\
& =L_{\text {coax }}-L_{a 1} .
\end{aligned}
$$

Now both $L_{\text {coax }}$ and $L_{a 1}$ depend on geometry only for wavelengths long as compared with their dimensions, and their values may thus be calculated. The values for $L_{\text {coax }}$ and $L_{a 1}$ are given by

$$
L=(2 \ln b / a) h \times 10^{-7},
$$

which is to be calculated for both $L_{\text {coax }}$ and $L_{a 1}$. The validity of this calibration constant over a wide range of frequencies, and turns ratios has been experimentally verified to better than one percent. Therefore, it is possible to calibrate the radio frequency permeameter, using physical dimensions only.

\section{Measurement of Temperature Coefficients of Permeability}

Kelsall originally designed an additional model of his permeameter to measure permeability coefficients of temperature [5]. This instrument is well suited for low frequencies but somewhat large for high-frequency use. A simple modification of the present instrument seemed to be more useful.

Figure 5 shows the modified permeameter for measurement of temperature coefficients of permeability. It is noted that except for an increase in the length of the secondary by the addition of cable the general structure of the instrument remains unchanged and so do the equations and measurement procedures. Because the value of $L_{2}$ has been changed, however, a different calibration constant will be obtained. In a convenient experimental setup a number of sample holders (the structure actually enclosing the test sample in fig. 6) are placed in the oven simultaneously. Each one has a cable of identical length leading to a coaxial switch. That portion of the permeameter containing the primary coil may then be connected to any one of the sample holders, one of which may be left empty to facilitate measurement of the short-circuited condition. Individual thermocouples can be connected to each sample to indicate its temperature.

The cables and connectors used in this work must, of course, be able to withstand high temperatures. Coaxial cable and connectors with Teflon dielectric are now available for this purpose and will operate satisfactorily up to $250^{\circ} \mathrm{C}$. The importance of this measurement is enhanced by the increasing need for core materials in military miniaturization work where temperatures up to $200^{\circ} \mathrm{C}$ are prevalent. This portion of the developmental work on the radiofrequency permeameter was sponsored by a miniaturization project at the National Bureau of Standards. 


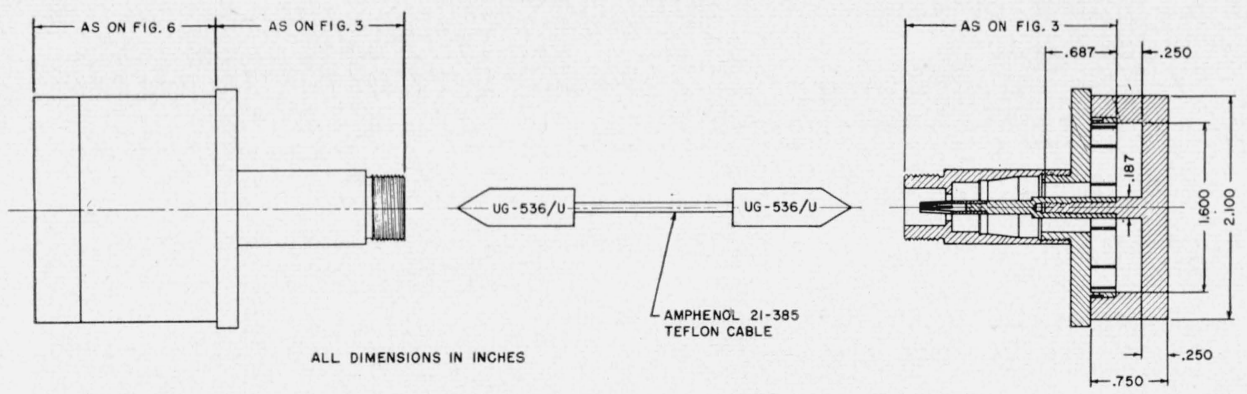

FIGURE 5. Modified radio-frequency permeameter for measuring temperature coefficient of samples.

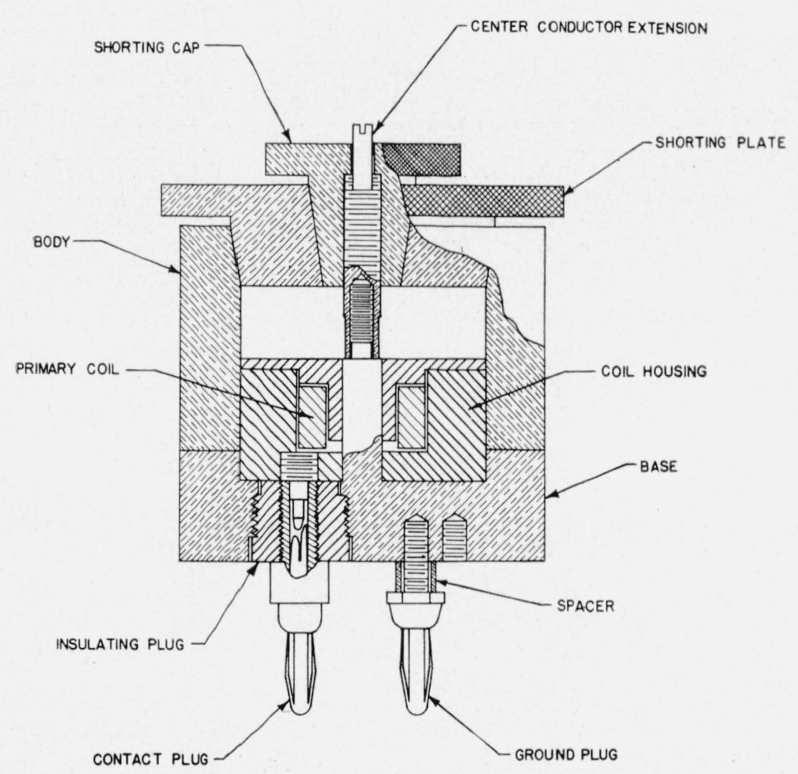

FiguRE 6. Cutaway view of radio-frequency permeameter.

The author is indebted to William A. Edson for suggesting the solution to the problem and to the Stackpole Carbon Co., St. Marys, Pa., for their cooperation in furnishing many Carbonyl powder toroids with extremely close tolerances in characteristics that are presently used as transformer cores in NBS permeameters. Figures 1 and 7 by courtesy of the National Electronic Laboratories.

\section{References}

[1] V. E. Legg, Magnetic measurements at low flux density using the alternating current bridge, Bell System Tech. J. 16, 39-62 (1936).

[2] Brockman, Dowling, and Steneck, Dimensional effects resulting from a high dielectric constant found in a ferromagnetic ferrite, Phys. Rev. 7 (January 1950).

[3] G. A. Kelsall, Permeameter for alternating current measurements at small magnetizing forces, J. Opt. Soc. Am. and Rev. Sci. Instr. 8 (February 1924).

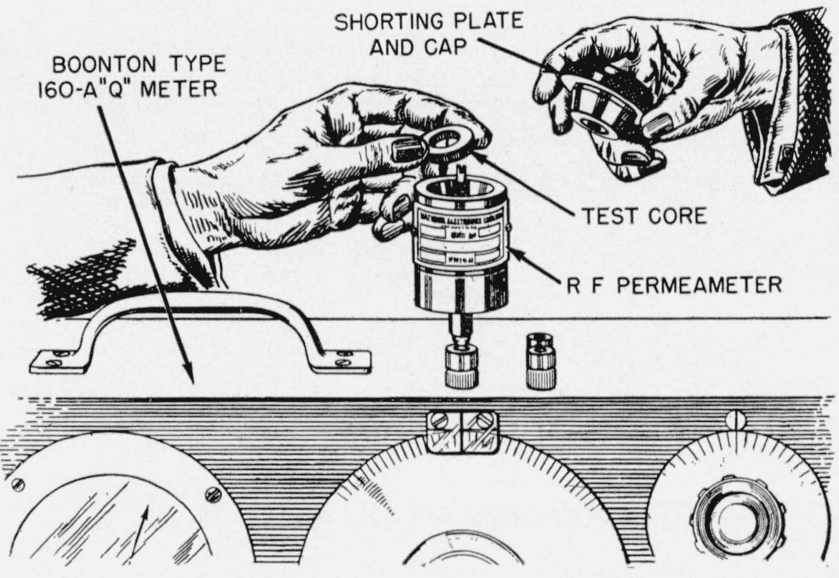

FiguRE 7. Radio-frequency permeameter in use with $Q$ meter.

[4] G. A. Kelsall, Permeameter for alternating current measurements at small magnetizing forces, J. Opt. Soc. Am. and Rev. Sci. Instr. 8, 334 (February 1924)

[5] G. A. Kelsall, Furnace permeameter for alternating current measurements at small magnetizing forces, J. Opt. Soc. Am. and Rev. Sci. Instr. 8 (May 1924).

\section{Appendix 1. Experimental Proof of the Validity of Equation 4}

Measurements were made on a particular model to show that the conditions for which eq (4) holds true can be met without difficulty.

These conditions are most difficult to satisfy at the lowest frequencies. The data given below were taken at $100 \mathrm{kc} / \mathrm{s}$ on a high-precision bridge.

From eq (2) and (10)

$R_{2}+j \omega L_{2}=\frac{\left(\frac{\omega L_{1}}{N}\right)^{2}\left(R_{0}-R_{1}\right)}{\left(R_{0}-R_{1}\right)^{2}+\omega^{2}\left(L_{0}-L_{1}\right)^{2}}+j \omega \frac{\left(\frac{\omega L_{1}}{N}\right)^{2}\left(L_{1}-L_{0}\right)}{\left(R_{0}-R_{1}^{2}\right)+\omega^{2}\left(L_{0}-L_{1}\right)^{2}}$

in which $N=100$ turns; $f=100$ kilocycles; $R_{0}=2.54$ ohms $R_{1}=1.46 \mathrm{ohm} ; L_{0}=50.59 \times 10^{-6}$ henry; and $L_{1}=81.56 \times 10^{-6}$ henry. Then, $R_{2}=2.87 \times 10^{-1} \mathrm{ohm} ; L_{2}=0.824 \times 10^{-8}$ henry ; and $\left(\omega L_{2} / R_{2}\right)^{2}=325$. 


\section{Appendix 2. Value of Equivalent Air Inductance}

The value of $L_{a}{ }^{5}$ is derived in Bureau of Standards Bulletin, Vol. 5, No. 3, p. 441, as

$$
L_{a}=\frac{4 K A}{10^{9} \mathrm{D}} \text { henry, }
$$

where $A$ is the cross-sectional area in square centimeters. The mean diameter $D=b+a, \quad K=1 / 2 p \ln (1+p) /(1-p)$, where $p=(b-a) /(b+a)$, the ratio of radial width to mean diameter, $b$ is the outside radius, and $a$ the inside radius.

Alternatively, an equivalent formula given in Bureau of Standards Circular 74, p. 251, eq (152), may be used:

$$
L_{a}=0.4606 h \log _{10} \frac{b}{a} \times 10^{-6} \text { henry, }
$$

where $h$ is the toroidal height in meters.

${ }^{5}$ This is the inductance due to the space having the dimensions of the test core.

Washington, September 2, 1953. 